\title{
Structural Analysis of Amino Acids, Oxidized by Reactive Oxygen Species and an Antibody against $N$-Formylkynurenine
}

\author{
Daisuke Suto $^{1,2}$, Yoshitaka Ikeda ${ }^{3}$, Junichi Fujiii,*, and Yoshihiro Ohba ${ }^{1}$ \\ ${ }^{1}$ Department of Chemical Engineering, Faculty of Engineering, Yamagata University, 4-3-16, Yonezawa 992-8510, \\ Japan \\ ${ }^{2}$ Department of Biomolecular Function, Graduate School of Medical Science, Yamagata University, \\ Yamagata 990-9585, Japan \\ ${ }^{3}$ Division of Molecular Cell Biology, Department of Biomolecular Sciences, Saga University Faculty of Medicine, \\ 5-1-1 Nabeshima, Saga 849-8501, Japan
}

Received 1 November, 2005; Accepted 30 November, 2005

\begin{abstract}
Summary Amino acid oxidation, resulting from oxidative stress, is related to certain diseases and aging. To correlate reactive oxygen species with oxidized products of individual amino acids, we oxidized Met, Trp, His, and Tyr by treatment with singlet oxygen, superoxide, or hydroxyl radicals. The structures of oxidized amino acids were determined by nuclear magnetic resonance spectroscopy, infra-red spectroscopy, and fast atom bombardment-mass spectrometry. Among the compounds identified, $N$-formylkynurenine (NFK), a well-known oxidation product of Trp in vivo via enzymatic and non-enzymatic reactions, and its hydroxylated form were found to be major products of the reaction with singlet oxygen. Since NFK is a pivotal oxidation product of Trp, we raised antiserum against NFK by immunizing a rabbit with NFK-conjugated bovine serum albumin. The resulting antiserum was then used to detect photooxidized trypsin and kynurenine-conjuated ovalbumin as well as NFK-albumin. An antiserum such as this would be useful tool for detecting NFK and kynurenine in situ.
\end{abstract}

Key Words: singlet oxygen, superoxide, hydroxyl radical, $N$-formylkynurenine, kynurenine

\section{Introduction}

Reactive oxygen species (ROS) are produced in vivo, in both enzymatic and non-enzymatic reactions, such as the Fenton reaction, and exert harmful effects on biological molecules, lipids, DNA, and proteins [1]. The involvement of ROS in skin photoaging has been extensively investigated [2]. The oxidative modification of proteins affects their function and appears to accelerate the aging process [3].

The oxidative modification of free amino acids and amino acid residues in proteins has been overviewed recently [4]. There appears to be correlation between generated ROS and certain oxidative modifications of individual amino

\footnotetext{
*To whom correspondence should be addressed.

Tel: +81-23-628-5227 Fax: +81-23-628-5230

E-mail: jfujii@med.id.yamagata-u.ac.jp
}

acids. Since hydroxyl radicals, generated by the Fenton reaction, are the most reactive among ROS, the oxidation products of amino acids by hydroxyl radicals have been the subject of extensive study. While most ROS are generated by electron transfer reactions, singlet oxygen is a non-radical species, generated by photochemical reactions of biological compounds, such as heme and flavins. Singlet oxygen is highly reactive with respect to certain biological components, especially those with resonance double bonds, such as polyunsaturated lipids [1].

Proteins contain numerous reactive residues and consume about $70 \%$ of the singlet oxygen by the reaction with them [5]. The sulfur atoms of Cys and Met are prone to oxidation and form different oxidation states. Glutathione, a tripeptidyl redox molecule, is present in relatively high levels in cells and functions as an antioxidant both by direct interaction with ROS and by donating electrons to glutathione peroxi- 
dases [6]. Upon oxidation under physiological conditions, the sulfhydryl group in glutathione is converted to either a sufenic/sulfinic acid or a disulfide. Cysteine residues in redox proteins, such as thioredoxin [7] and peroxiredoxin [8], are highly reactive, and their functions have also been investigated from the viewpoint of physiology and diseases [9]. On the other hand, Trp is oxidatively converted to kynurenine and other physiologically important compounds via both enzymatic and non-enzymatic pathways $[10,11]$.

Here we report on a comparison of structures and yields of derivatives of four amino acids, Trp, His, Tyr, and Met that are produced by oxidation by singlet oxygen, superoxide, or hydroxyl radicals. We also raised an antibody against $N$ formylkynurenine (NFK), a major product produced from Trp oxidation by singlet oxygen.

\section{Materials and Methods}

All reagents used in this study were the highest grade available. To protect against oxidation, the $\alpha$-amino group of Trp, His, Tyr, and Met was blocked by the addition of a $t$ butyloxycarbonyl (Boc) group. Each amino acid was oxidatively modified by reaction with either singlet oxygen, superoxide, or hydroxyl radicals as follows.

\section{Generation of singlet oxygen}

Singlet oxygen was generated by two methods. One involves a photosensitization reaction in the presence of 36 $\mu \mathrm{M}$ methylene blue (MB) at $15-18^{\circ} \mathrm{C}$ for $6.5 \mathrm{~h}$ in water [12]. The other involves the chemical reaction of $3-4.4 \mathrm{mM}$ $\mathrm{Na}_{2} \mathrm{MoO}_{4}$ and $26.1-53 \mathrm{mM} \mathrm{H}_{2} \mathrm{O}_{2}$ at $20^{\circ} \mathrm{C}$ for $45-75 \mathrm{~min}$.

\section{Generation of superoxide}

Superoxide was generated by the reaction of $1.5-2.4 \mathrm{mM}$ $\mathrm{KO}_{2}$ and $0.19-0.3 \mathrm{mM} 18$-crown-6-ether in dry acetonitrile in air at $-10^{\circ} \mathrm{C}$ for $5-7 \mathrm{~h}[13]$.

\section{Generation of hydroxyl radicals}

Hydroxyl radicals were generated by the Fenton reaction in the presence of $0.2-0.4 \mathrm{mM} \mathrm{FeSO}_{4}, 0.2-0.4 \mathrm{mM}$ diethylenetriamine pentaacetic acid (DTPA), and $2-4 \mathrm{mM} \mathrm{H}_{2} \mathrm{O}_{2}$ at $20^{\circ} \mathrm{C}$ for $30-45 \mathrm{~min}$.

\section{Purification and structural analysis of the products}

After extraction and washing, the products were purified by chromatography on columns of Kiesel 60 (Merck, Darmstadt, Germany) or Silicagel 60 F 254 (Merck, Darmstadt, Germany). Some of the compounds were further purified by crystallization. The structures of the products were determined by ${ }^{1} \mathrm{H}$ - and ${ }^{13} \mathrm{C}$-nuclear magnetic resonance (NMR), infra-red (IR) spectroscopy, and fast atom bombardmentmass spectrometry (FAB-MS).

\section{Conjugation and raising antibody}

BocNFK and kynurenine (Sigma, Tokyo, Japan) were conjugated to bovine serum albumin (BSA) (Wako, Osaka, Japan) and ovalbumin (OVA) (Wako, Osaka, Japan) respectively. After conjugation to BSA, the Boc group was removed by hydrolysis with aqueous trifluoroacetic acid (Wako, Osaka, Japan). NFK-conjugated BSA was used for immunization of a female rabbit. Trypsin (Sigma, Tokyo, Japan) was subjected to photooxidation in the presence of MB.

\section{SDS-PAGE and immunoblot analysis}

Protein samples were subjected to $12 \%$ SDS-PAGE and then transferred to a Hypond-P membrane (Amersham Pharmacia Biotech, Buckinghamshire, UK) under semi-dry conditions by means of a Transfer-blot SD Semi-dry transfer cell (Bio-Rad, Hercules, USA). The membrane was then blocked by incubation with $5 \%$ skimmed milk in Trisbuffered saline (TBS; $150 \mathrm{mM} \mathrm{NaCl}$ and $20 \mathrm{mM}$ Tris- $\mathrm{HCl}$, $\mathrm{pH}$ 7.6) for $2 \mathrm{hr}$ at room temperature. The membranes were then incubated with the rabbit antiserum against NFKconjugated BSA (1:50 dilution) for $16 \mathrm{~h}$ at $4^{\circ} \mathrm{C}$. After washing with TBS containing $0.1 \%$ Tween-20, the membrane was incubated with $1: 1,000$ diluted peroxidaseconjugated goat anti-rabbit IgG (Santa Cruz Biotechnology, Santa Cruz, USA) for $1 \mathrm{~h}$ at room temperature. After washing, the peroxidase activity on the membranes was detected by a chemiluminescence method using an ECL Plus kit (Amersham Pharmacia Biotech, Buckinghamshire, UK) and exposed to X-ray films (Kodak, Rochester, USA).

\section{Results}

\section{Structures and yields of oxidation products}

Since proteins contain numerous amino acids in different environments, it is more useful to use free amino acids to identify oxidation products from an individual amino acid. We chose four amino acids, Trp, His, Tyr, and Met, in which their amino groups were blocked with a Boc group, and oxidized them by treatment with singlet oxygen, superoxide, or hydroxyl radicals. After purification by column chromatography, the structures of the oxidation products were determined by ${ }^{1} \mathrm{H}$ - and ${ }^{13} \mathrm{C}-\mathrm{NMR}$, IR, and FAB-MS.

Fig. 1. summarizes structure of the products and their yields. The oxidation of Met by singlet oxygen produced a sulfonate derivative $(45 \%)$, while hydroxymethionine was produced by reaction with hydroxyl radicals under these conditions. The reaction of Trp with singlet oxygen generated both NFK and hydroxy-NFK. The oxidation of His resulted in a complex mixture of compounds. Oxygen was added to the imidazole ring by reaction with singlet oxygen. The reaction of His with superoxide generated a mixture of two compounds with translocated groups. We 


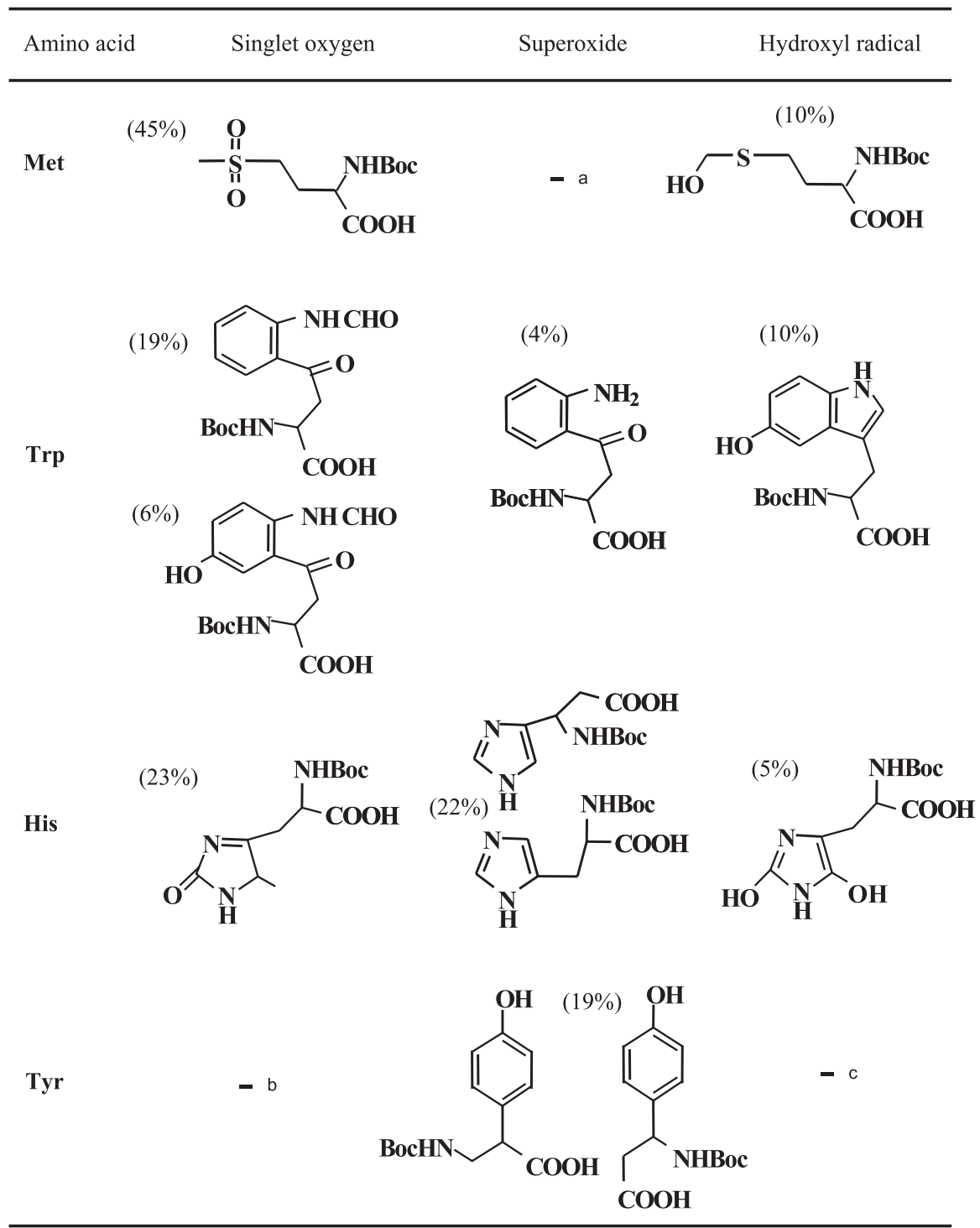

a Not examined

b,c Not identified

Fig. 1. Structures and yields of products produced from Trp, His, Tyr, and Met by oxidation with singlet oxygen, superoxide, or hydrogen peroxide. Structures of the oxidation products of each amino acid and their yields are shown. Since the reaction of Tyr with singlet oxygen or hydroxyl radicals generated numerous break down products, it was impossible to isolate them in pure form. The oxidation of Met with superoxide was not examined.

were unable to isolate reaction products of Tyr with superoxide or hydroxl radicals because Tyr was extensively destroyed under the conditions used, while the reaction with superoxide induced group translocation.

Generation and characterization of antiserum against NFK

Since the oxidation of Trp with singlet oxygen produces NFK, which is generated during the oxidative metabolism of Trp [11] and also found in oxidized proteins [3], we attempted to produce antibody against it. A rabbit was immunized by subcutaneous injection of NFK-conjugated BSA. As a reference sample, trypsin was photooxidized in the presence of MB and subjected to an immunoblot analysis using this antiserum. As a result, the antiserum reacted with photooxidized trypsin, but not an untreated sample (Fig. 2A). The reactivity of the antiserum increased in a photooxidation-time dependent manner, suggesting that the epitope is generated during the photooxidation of the protein. Since kynurenine is predominantly present in the body, the reactivity to kynurenine was also examined (Fig. 2B). When kynurenine-conjugated OVA was subjected to an immunoblot analysis, the antiserum also detected kynurenine-conjugated OVA but not control OVA. The presence of $0.5 \mathrm{mg} / \mathrm{ml}$ kynurenine-conjugated OVA during 


\section{(A) Photooxidized Trypsin}

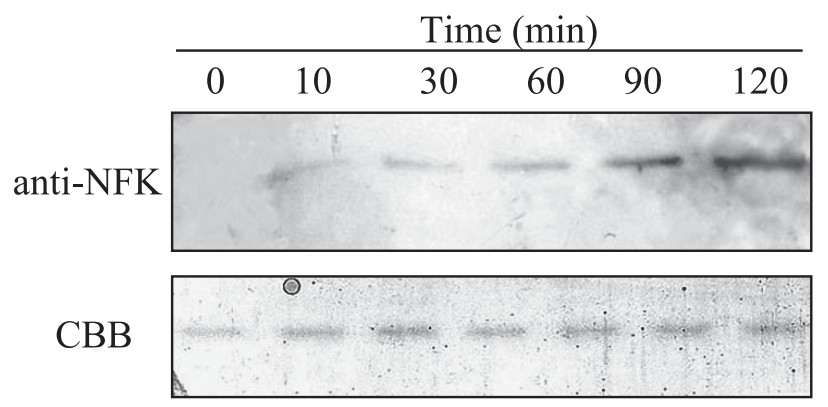

\section{(B) Kynurenine-OVA}

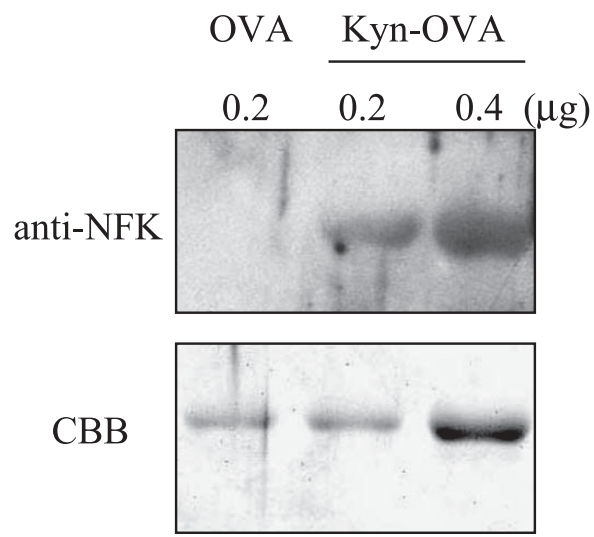

Fig. 2. Characteristics of the antiserum raised against NFK. (A) Trypsin was photooxidized for the indicated times, separated by SDS-PAGE, and blotted onto a nylon membrane. An immunoblot analysis was performed on the membrane with anti-NFK antiserum (1:50 dilution) as a primary antibody. (B) Kynurenine-conugated OVA (Kyn-OVA) was subjected to the immunoblot analysis with the same antiserum as above.

incubation suppressed the binding of the antibody (data not shown). Thus, the antiserum was able to recognize both the NFK and kynurenine epitopes. This is the first report of the production of antiserum against NFK and kinurenine on proteins.

\section{Discussion}

To understand the correlation between oxidative stress and disease, it is necessary to be in a position to specify which type of ROS is responsible for the oxidation of a specific cellular component. We initially selected four amino acids for this purpose because they are sensitive to oxidation and, physiologically, play important roles. Among the ROS, singlet oxygen effectively oxidized Trp under these conditions (Fig. 1). Singlet oxygen was found to react with other amino acids rapidly and oxidatively modified them, as reported previously [14]. A reaction scheme for NFK production by the oxidation of Trp with singlet oxygen has been postulated [15].

Under severe oxidative stress, ROS are produced in large amounts, induce irreversible damages to cellular components, and trigger apoptosis [16, 17]. At the initial stage of apoptosis after the stimuli, cytochrome $\mathrm{c}$ is generally released from mitochondria and activates apoptosome formation, leading to the activation of the caspase cascade. We have recently shown that the cell death induced by singlet oxygen is atypical to apoptosis [18]. This is due to the suppression of the caspase cascade prior to apoptosome formation and the direct inhibition of caspase activity and the proapoptotic function of cytochrome $\mathrm{c}$ by singlet oxygen $[18,19]$. Despite extensive study, however, we were unable to identify the amino acid modification responsible for this, probably because of the complex nature of the oxidation products.

Recent advances in proteomic studies using mass spectrometry and a computer data base permit the systematic profiling of thousands of proteins in a short time [20]. However, identification of oxidative modification of individual amino acids in vivo during pathogenesis and aging is still quite difficult and time-consuming. This is partly due to the divergence and heterogeneity of the oxidized amino acids in a whole protein. In vivo, oxidative stress causes disulfide formation and the sulfoxidation of Cys and/or Met as initial reaction products. Since glutathione is present at millimolar concentrations in most cells, sulfhydryl group of glutathione can be readily oxidized, forming glutathione disulfide or a mixed disulfide with proteins. We recently established a simple system for determining glutathionylated protein using glutathione S-transferase as a probe molecule [21]. The oxidation of other amino acids also occurs and their contents increase during the aging process [3]. Since, in general, carbonyl groups are frequently introduced into oxidized amino acids, protein carbonyl content is measured as a marker of protein oxidation. However, a tool for separately detecting the oxidation products of an individual amino acid is not currently available. In this regard, specific antibodies that recognize each oxidation product would be useful for their detection in situ and would facilitate our understanding of the causal connection between amino acid oxidation and the corresponding disease.

Hence, we established an antiserum against NFK that recognizes oxidized protein (Fig. 2). The oxidative metabolism of Trp has physiologically important roles. Kynurenine is formed from Trp by the action of indolamine 2,3-dioxygenase and is further metabolized via the kynurenine pathway to various phenolic compounds [10]. NFK is produced from Trp by the dioxygenase reaction and is rapidly 
hydrolyzed into kynurenine by a kynurenine formamidase [11]. Our data show that NFK is predominantly generated by reaction with singlet oxygen. Once released from proteins, NFK generated by the non-enzymatic reaction would also enter into the kynurenine pathway and be further metabolized. On the other hand, some oxidation products of amino acids are still reactive and are involved in other biological functions. While protein-bound kynurenine functions as a photosensitizer and generates additional singlet oxygen [22], some Trp metabolites including kynurenine appear to have antioxidant properties [23].

The production of NFK as well as other oxidized amino acids was reported to be increased in affected brain lesions of Alzheimer disease [24]. However, to quantify the individual oxidation products, the development of more advanced technology is awaited [25]. The data presented here suggest that the antiserum recognizes NFK on an oxidized protein as well as kynurenine-bound proteins. The use of this antibody would facilitate detection of NFK on proteins in a conventional laboratory and lead to the development of an understanding of the roles of protein oxidation in diseases and aging.

\section{Acknowledgments}

This work was supported, in part, by the Cosmetology Research Foundation.

\section{References}

[1] Halliwell, B. and Gutteridge, J.M.C.: Free Radicals in Biology and Medicine 3rd ed. Oxford: Oxford Science, 1-35, 1998.

[2] Briviba, K., Klotz, L.O., and Sies, H.: Toxic and signaling effects of photochemically or chemically generated singlet oxygen in biological systems. Biol. Chem., 378, 1259-1265, 1997.

[3] Berlett, B.S. and Stadtman, E.R.: Protein oxidation in aging, disease, and oxidative stress. J. Biol. Chem., 272, 2031320316, 1997.

[4] Stadtman, E.R. and Levine, R.L.: Free radical-mediated oxidation of free amino acids and amino acid residues in proteins. Amino Acids, 25, 207-218, 2003.

[5] Davies, M.J.: The oxidative environment and protein damage. Biochim. Biophys. Acta., 1703, 93-109, 2005.

[6] Meister, A.: Glutathione biosynthesis and its inhibition. Methods Enzymol., 252, 26-30, 1995.

[7] Nakamura, H., Nakamura, K., and Yodoi, J.: Redox regulation of cellular activation. Annu. Rev. Immunol., 15, 351-369, 1997.

[8] Fujii, J. and Ikeda, Y.: Advances in our understanding of peroxiredoxin, a multifunctional, mammalian redox protein. Redox Rep., 7, 123-130, 2002.

[9] Claiborne, A.L., Mallett, T.C., Yeh, J.I., Luba, J., and Parsonage, D.: Structural, redox, and mechanistic prameters for cysteine-sulfenic acid function in catalysis and regulation. Adv. Protein Chem., 58, 215-276, 2001.

[10] Fujiwara, M., Shibata, M., Watanabe, Y., Nukiwa, T., Hirata, F., Mizuno, N., and Hayaishi, O.: Indoleamine 2,3-dioxygenase. Formation of L-kynurenine from L-tryptophan in cultured rabbit fineal gland. J. Biol. Chem., 253, 6081-6085, 1978.

[11] Takikawa, O., Yoshida, R., Kido, R., and Hayaishi, O.: Tryptophan degradation in mice initiated by indoleamine 2,3dioxygenase. J. Biol. Chem., 261, 3648-3653, 1986.

[12] Savive, W.E.: Isolation and identification of some photooxidation products of tryptophan. Aust. J. Chem., 24, 12851293, 1971.

[13] Ito, T., Nagata, K., Okada, M., and Ohsawa, M.: Redox properties of superoxide with 1,2,3-triazine derivatives. Tetrahedron Lett., 31, 7193-7196, 1990.

[14] Michaeli, A. and Feitelson, J.: Reactivity of singlet oxygen toward amino acids and peptides. Photochem. Photobiol., 59, 284-289, 1994.

[15] Weil, L., Gordon, W.G., and Buchert, A.R.: Photooxidation of amino acids in the presence of methylene blue. Arch. Biochem., 33, 90-109, 1951.

[16] Green, D. and Reed, J.C.: Mitochondria and apoptosis. Science, 281, 1309-1312, 1998.

[17] Hengartner, M.O.: The biochemistry of apoptosis. Nature, 407, 770-776, 2000.

[18] Otsu, K., Sato, K., Ikeda, Y., Ohba, Y., and Fujii, J.: An abortive apoptotic pathway induced by singlet oxygen is due to the suppression of caspase activation. Biochem. J., $\mathbf{3 8 9}$, 197-206, 2005.

[19] Suto, D., Sato, K., Ohba, Y., Yoshimura, T., and Fujii, J.: Suppression of the pro-apoptotic function of cytochrome $\mathrm{c}$ by singlet oxygen via a heme redox state-independent mechanism. Biochem. J., 392, 399-406, 2005.

[20] Guan, J.Q. and Chance, M.R.: Structural proteomics of macromolecular assemblies using oxidative footprinting and mass spectrometry. Trends Biochem. Sci., 30, 583-592, 2005.

[21] Cheng, G., Ikeda, Y., Iuchi, Y., and Fujii, J.: Detection of S-glutathionylated proteins by glutathione S-transferase overlay. Arch. Biochem. Biophys., 435, 42-49, 2005.

[22] Parker, N.R., Jamie, J.F., Davies, M.J., and Truscott, R.J.: Protein-bound kynurenine is a photosensitizer of oxidative damage. Free Radic. Biol. Med., 37, 1479-1489, 2004.

[23] Christen, S., Peterhans, E., and Stocker, R.: Antioxidant activities of some tryptophan metabolites: possible implication for inflammatory diseases. Proc. Natl. Acad. Sci. USA., 87, 2506-2510, 1990.

[24] Ahmed, N., Ahmed, U., Thornalley, P.J., Hager, K., Fleischer, G., and Munch, G.: Protein glycation, oxidation and nitration adduct residues and free adducts of cerebrospinal fluid in Alzheimer's disease and link to cognitive impairment. J. Neurochem., 92, 255-263, 2005.

[25] Domingues, M.R., Domingues, P., Reis, A., Fonseca, C., Amado, F.M., and Ferrer-Correia, A.J.: Identification of oxidation products and free radicals of tryptophan by mass spectrometry. J. Am. Soc. Mass Spectrom., 14, 406-416, 2003. 